\title{
A Comparative Study on the Corrosion Behaviour of Hard Anodic Coatings on AA 6061 Obtained Using DC and Pulsed DC Power Sources
}

\author{
Franco M., Hari Krishna T., Anju M. Pillai ${ }^{\dagger}$, A. Rajendra and A.K. Sharma \\ Thermal Systems Group, ISRO Satellite Centre, Vimanapura Post, Bangalore 560017, India \\ [Manuscript received 13 February 2013, in revised form 07 May form 2013] \\ (C) The Chinese Society for Metals and Springer-Verlag Berlin Heidelberg
}

\begin{abstract}
The corrosion behaviour of the hard anodic coatings prepared by three different methods viz., conventional hard anodizing (C-HA), pulse hard anodizing (P-HA) and low voltage room temperature pulse hard anodizing (LVPHA) on AA 6061 was compared using electrochemical impedance spectroscopy (EIS) and linear polarisation. Scanning electron microscope (SEM) was used to study the surface morphology before and after corrosion. EIS data revealed that no significant difference in corrosion resistance was observed among three types. $R_{\mathrm{p}}$, $I_{\text {corr }}$ and $E_{\text {corr }}$ were found to be highly stable over the exposure period up to $72 \mathrm{~h}$ which confirms the excellent corrosion resistance. The studies indicate that the corrosion resistance of LVP-HA and P-HA, which are processed at lower voltage and at relatively higher temperatures, are comparable to that of C-HA which is processed at sub-zero temperatures and high operating voltages. The corrosion resistance of all coatings was found to be improved after hydrothermal sealing.
\end{abstract}

KEY WORDS: Aluminium; Anodic films; EIS; Polarisation; SEM

\section{Introduction}

Aluminium and its alloys have tremendous application in the field of space technology and automotive industries. This wide usage is due to their excellent properties, such as high strength-to-weight ratio and good formability. Unfortunately, aluminium alloys are susceptible to corrosion which limits their applications ${ }^{[1]}$. Though formation of a natural oxide layer on its surface which is about $2.5 \mathrm{~nm}^{[2]}$ can slightly prevent corrosion, degradation occurs on prolonged storage. Pitting corrosion is the most common type of corrosion encountered in these alloys. In order to improve the corrosion resistance, surface treatments such as anodising can be employed ${ }^{[2-4]}$.

Anodising, which produces an outer porous array of columnar hexagonal cells normal to the substrate

† Corresponding author. Tel.: +91-80-25037375; Fax: +91-8025083203; E-mail address: anjum@isac.gov.in(Anju M. Pillai)

DOI: $10.1007 / \mathrm{s} 40195-013-0091-2$ surface and separated from it by a barrier layer ${ }^{[5]}$, can offer high corrosion resistance ${ }^{[6,7]}$. Conventional anodising of aluminium can build the coating of moderate thickness of $1.8 \mu \mathrm{m}$ to $25 \mu \mathrm{m}$ and are known as Type II, as classified by MIL-A-8625, while coatings thicker than $25 \mu \mathrm{m}$ are known as Type III, hard coat, hard anodising, or engineered anodising. Anodising thickness increases wear resistance, corrosion resistance, electrical and thermal insulation etc. ${ }^{[8]}$. The hard anodised coatings are of paramount importance in engineering industries where high wear resistant and corrosion resistant surfaces are required such as pistons, cylinders, hydraulic gears etc. ${ }^{[9,10]}$. Hard anodisation process has also been used in fabrication of sensors due to its insulating properties ${ }^{[11]}$.

Conventional hard anodising process, which is carried out with dc power supply, requires the processing bath to be controlled at sub-zero temperature in which enormous electrical energy has to be supplied for the refrigeration ${ }^{[10,11]}$. Moreover it is associated with the problems like burning and powdering due to low temperature processing and utilization of high 
Franco M. et al.: Acta Metall. Sin. (Engl. Lett.), 2013, 26(6), 647-656.

Table 1 Processing parameters of the all three samples

\begin{tabular}{cccccc}
\hline Process & $\begin{array}{c}\text { Temperature } \\
\left({ }^{\circ} \mathrm{C}\right)\end{array}$ & $\begin{array}{c}\text { Current density } \\
\left(\mathrm{A} / \mathrm{cm}^{2}\right)\end{array}$ & $\begin{array}{c}\text { Duty cycle } \\
(\%)\end{array}$ & $\begin{array}{c}\text { Frequency } \\
(\mathrm{Hz})\end{array}$ & $\begin{array}{c}\text { Processing time } \\
(\mathrm{min})\end{array}$ \\
\hline C-HA & $-5 \pm 2$ & $325 \pm 10$ & - & - & $80-100$ \\
P-HA & $10 \pm 2$ & $540 \pm 10$ & $60-75$ & $5-10$ & $50-60$ \\
LVP-HA & $25 \pm 2$ & $645 \pm 10$ & $60-80$ (forward), & $10-20$ (forward), & $40-60$ \\
& & $60-80$ (reverse) & $400-500$ (reverse) \\
\hline
\end{tabular}

Note: Processing time varied to get a coating thickness of $(60 \pm 3) \mu \mathrm{m}$; the electrolyte is $100 \mathrm{~mL} / \mathrm{L} \mathrm{H}_{2} \mathrm{SO}_{4}+15 \mathrm{~g} / \mathrm{L} \mathrm{C}_{2} \mathrm{H}_{2} \mathrm{O}_{4}$

current density. Pulse hard anodising and low voltage room temperature pulse hard anodising, which are processed using DC pulse and pulsed DC with reverse pulse techniques respectively, are carried out at elevated temperatures and therefore these are cost effective. Moreover, the chances of burning is minimised because the voltage applied in pulse and reverse pulse hard anodising is lower compared to conventional hard anodising ${ }^{[11]}$.

Generally, the corrosion resistance of an anodic film depends on the properties, thickness and integrity of the film. The sealing quality is one of the factors affecting the film properties. The sealing treatment in general enhances the corrosion resistance and the electrical insulating value of the anodic film. Different sealing techniques for the anodic film on aluminium alloys were developed by many researchers. Some sealing techniques have been commonly used in industry, such as hot water sealing, steam sealing, dichromate sealing, nickel acetate sealing and cold nickel fluoride sealing. Boiling water sealing is the simplest and effective one which can prevent the risk of pitting corrosion even in most aggressive atmosphere ${ }^{[12-15]}$.

In recent years electrochemical impedance spectroscopy (EIS) has become a powerful technique to evaluate the electrochemical behaviour of electrode - electrolyte interfaces. In this technique, a small AC amplitude signal is applied to the system being studied. Therefore, EIS is a non-destructive technique that does not affect the electrode properties to be measured and is suitable for in-situ monitoring of many corrosion processes that occur in wide range of materials, including metallic coatings, anodic coatings and corrosion inhibitors ${ }^{[3,12,13,16-20]}$. The corrosion behaviour of anodised layer during exposure to corrosive medium can also be studied as a function of exposure time by $\operatorname{EIS}^{[1-3]}$.

In the present work, the corrosion behaviour of the three different types of hard anodising processes viz., conventional hard anodising (C-HA), pulse hard anodising (P-HA) and low voltage room temperature pulse hard anodising (LVP-HA) were investigated using EIS and polarisation techniques. The main aim of the work was to check the comparability of corrosion behaviour of LVP-HA and P-HA with C-HA so as to make the two former processes an alternative to the latter. Scanning electron microscopy was used to study the surface morphology of the coatings before and after corrosion.

\section{Experimental details}

\subsection{Materials}

Aluminium alloy $6061 \mathrm{~T} 6$ specimens (composition (wt.\%): Mg 0.8-1.2, Si 0.6, Cu 0.28, Mn 0.15, Fe 0.7 and remainder $\mathrm{Al}$ balanced) of diameter $24 \mathrm{~mm}$ and thickness $4 \mathrm{~mm}$ were used in the present study.

\subsection{Preparation of hard anodised specimens}

The specimens were subjected to cleaning (pretreatment) before anodisation as per the following sequence.

Ultra sonic cleaning using trichloroethylene at room temperature for 3-5 min.

Alkaline cleaning in the solution with $20-30 \mathrm{~g} / \mathrm{L}$ $\mathrm{Na}_{2} \mathrm{CO}_{3}, 25-35 \mathrm{~g} / \mathrm{L}$ Tri sodium ortho-phosphate, 15$25 \mathrm{~g} / \mathrm{L}$ sodium meta silicate and $1 \mathrm{~g} / \mathrm{L}$ wetting agent prepared in de-mineralised (DM) water. Cleaning was done using mild agitation for $3-5 \mathrm{~min}$ at $(60 \pm 5){ }^{\circ} \mathrm{C}$ followed by DM water rinsing.

Acid de-smutting in the solution with10-15 mL/L $\mathrm{H}_{2} \mathrm{SO}_{4}, 10-12 \mathrm{~mL} / \mathrm{L} \mathrm{HF}$ and $20-25 \mathrm{~mL} / \mathrm{L} \mathrm{HNO}_{3}$ at $20-25{ }^{\circ} \mathrm{C}$ for $2-3 \mathrm{~min}$. This was followed by thorough rinsing in DM water.

Hard anodising process was carried out in a lead lined tank which was made as cathode and job as anode. Air agitation was given to the bath with continuous filtration. The process parameters are summarised in Table 1. After hard anodisation some of the samples were subjected to hydrothermal sealing in boiling water for about 30 min to study the effect of sealing on corrosion performance.

\subsection{Electrochemical Impedance tests}

Corrosion behaviour of the three different hard anodised specimens was monitored using electrochemical impedance spectroscopy (EIS). It was performed using a frequency response analyser (Autolab PGSTAT 302N, Eco-chemie, The Netherlands) driven by Nova 1.4 software. A typical three electrodes cell, $\mathrm{Ag} / \mathrm{AgCl}$ (saturated with $\mathrm{KCl}$ ) as reference electrode, a platinum rod as counter electrode and the specimen as the working electrode $\left(1 \mathrm{~cm}^{2}\right.$ exposed area) were used in the cell. All the electrochemical tests were conducted in an unstirred, neutral $3.5 \mathrm{wt} . \% \mathrm{NaCl}$ so- 

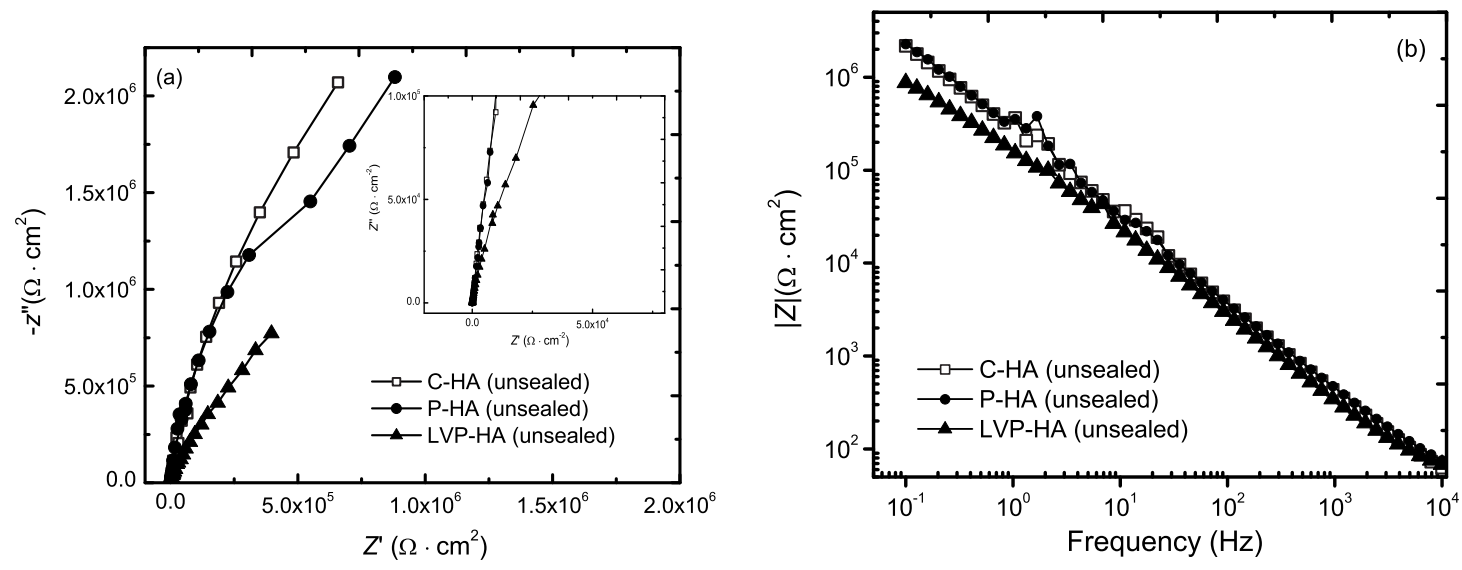

Fig. 1 Nyquist diagram (a) and Bode plots (b) for unsealed hard anodised samples after $24 \mathrm{~h}$ of immersion in 3.5 wt.\% $\mathrm{NaCl}$ solution

lution at room temperature. Prior to test, the hard anodised samples (both sealed and unsealed condition) were subjected to 24,48 and $72 \mathrm{~h}$ of immersion in 3.5 wt.\% $\mathrm{NaCl}$ solution to simulate the prolonged/accelerated exposure. The EIS tests were performed with a sinusoidal AC amplitude signal of $10 \mathrm{mV}$ over the open circuit potential $\left(E_{\text {ocp }}\right)$ in the frequency range from $10 \mathrm{kHz}$ to $0.1 \mathrm{~Hz}$.

\subsection{Polarisation tests}

To confirm the corrosion results of hard anodic coatings obtained by EIS, polarisation tests were also conducted on test specimens using Nova 1.4 software in the same instrument (Autolab PGSTAT 302N, potentiostat/galvanostat, The Netherlands). The tests were performed at a scan rate of $0.1 \mathrm{mV} / \mathrm{s}$ in the applied potential range of $-0.250 \mathrm{~V}$ to $+0.250 \mathrm{~V}$ with respect to $E_{\text {corr }}$.

\subsection{Surface morphology and thickness measurement}

The corrosion morphology of the test samples was examined by a scanning electron microscope (LEICA S. 400 I, CAMBRIDGE, UK), and the extent of corrosion after immersion in the corrosive medium was analysed.

Coating thickness measurement was carried out with Fischer ISOSCOPE thickness meter which work on the principle of eddy current.

\section{Results and Discussion}

\subsection{EIS results for the hard anodic coatings without sealing}

The most common and standard representation of impedance spectroscopy are Nyquist and Bode plots. The impedance curve of real and imaginary parts is illustrated in Nyquist diagram. Larger the diameter better is the impedance or resistance to corrosion. In
Bode plots, modulus of impedance and phase shift are plotted as a function of frequency over a wide frequency range. The higher intercept of the values of modulus of impedance $(|Z|)$ on $y$-axis shows better resistance towards corrosion. Thus it is from this resulting structure of $Z(\omega)$ vs. frequency response that one derives information about electrical properties of full electrode-material system ${ }^{[21]}$.

The Nyquist and Bode plots pertaining to the three unsealed hard anodised samples (C-HA, P$\mathrm{HA}$ and LVP-HA) subjected to $24 \mathrm{~h}$ immersions in 3.5 wt. $\% \mathrm{NaCl}$ solution are shown in Fig. 1(a) and (b). Very high impedance curves are observed for all three types of hard anodic coatings. The dimension of the capacitive characteristic curve for C-HA is slightly greater as compared to that of P-HA and LVP-HA samples. Thus the corrosion resistance of the hard anodic coatings decreases in the order C-HA $>$ P-HA > LVP-HA. This could be due to the difference in the size and density of the pores among three types of samples. One of the reasons for porosity difference is the solvation effect of the electrolyte. This effect is lower in C-HA than those of P-HA and LVP-HA. However, there is no significant difference in $R_{\mathrm{p}}$ values of three samples i.e., the values are all in same order $\left(10^{6} \Omega \cdot \mathrm{cm}^{2}\right)$ as summarised in Table 2 . Higher $R_{\mathrm{p}}$ value represents better corrosion resistance $^{[22]}$. $R_{\mathrm{p}}$ values exceeding $2 \times 10^{5} \cdot \Omega \mathrm{cm}^{2}$ are considered to be properly anodised samples for common applications ${ }^{[23]}$. Absence of inductive loop suggests that no localized corrosion has taken place ${ }^{[24]}$. The high impedance towards low frequency is characteristic of good corrosion resistance ${ }^{[25,26]}$. From the Bode plot in Fig. 1(b), it is clear that higher impedance values are observed on approaching lower frequency which indicates that the coating is highly compact and no major penetration of the corrosive medium has taken place. The advantage of Bode plot is that the data for all the measured frequencies are shown and also a wide range of impedance values are displayed. In the Bode diagram the values of $|Z|$ and 
Table 2 EIS data of hard anodic coatings after immersion in 3.5 wt.\% $\mathrm{NaCl}$ solution

\begin{tabular}{cccccccc}
\hline Immersion time $(\mathrm{h})$ & \multicolumn{3}{c}{ CPE $\left(10^{-7} \mathrm{~F} / \mathrm{cm}^{2}\right)$} & & \multicolumn{3}{c}{$R_{\mathrm{p}}\left(10^{6} \Omega \cdot \mathrm{cm}^{2}\right)$} \\
\cline { 2 - 4 } \cline { 7 - 8 } & C-HA & P-HA & LVP-HA & & C-HA & P-HA & LVP-HA \\
\hline 24 & $1.48(0.322)$ & $2.07(0.586)$ & $3.42(0.83)$ & & $10.7(49.5)$ & $7.6(27.16)$ & $4.6(19.18)$ \\
48 & $2.11(0.488)$ & $3.08(0.819)$ & $3.84(1.11)$ & & $7.5(32.6)$ & $5.16(19.4)$ & $4.1(14.3)$ \\
72 & $3.07(0.848)$ & $3.51(1.434)$ & $4.59(2.365)$ & & $5.1(18.77)$ & $4.5(11.1)$ & $3.5(6.7)$ \\
\hline
\end{tabular}

Note: values in the brackets are for the sealed speciments

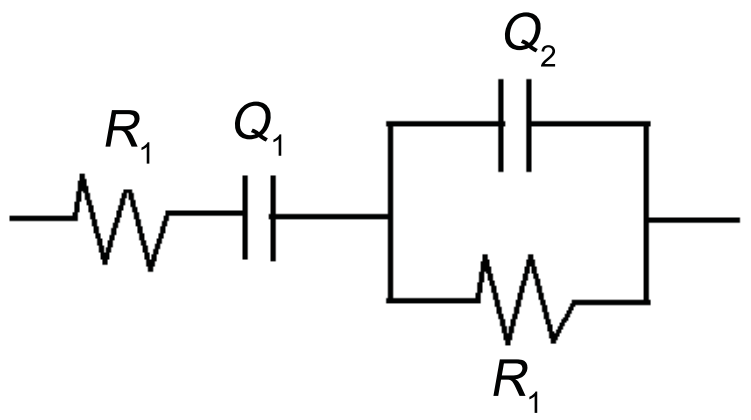

Fig. 2 A possible equivalent circuit for hard anodised coating for aluminium alloys

frequency are plotted in the logarithmic scale. The impedance curves from Nyquist plot and Bode plots show the typical curve for highly corrosion resistant coating where no Warburg or diffusion control reaction has taken place. Hence, it can be concluded that there is no significant deterioration in initial stage of immersion (up to $24 \mathrm{~h}$ ) in aggressive medium $(3.5$ wt. $\% \mathrm{NaCl})$.

The possible equivalent circuit for this type of the hard anodic coatings can be proposed as $\left[R_{1} Q_{1}\left(R_{2} Q_{2}\right)\right]$ in circuit description code $(\mathrm{CDC})$ in which elements within the bracket are connected in parallel. The equivalent circuit which gives the best fitting for all the plots is shown in Fig. 2. $R_{\mathrm{p}}$ values obtained from the Bode plots at the least frequency $(0.1 \mathrm{~Hz})$ are approximately same as that observed in the electrochemical circle fit.

Since the outer layer is much thicker than inner barrier layer for hard anodic coatings, the capacitive characteristic line (inset in Fig. 1(a)) which is nearly normal to $Z_{\text {re }}$ axis is observed at high frequency region in Nyquist plots (Fig. 1(a)). This region is simulated as $Q_{1}$, constant phase element (CPE), for thicker outer layer. $R_{1}$ represents the solution resistance. $Q_{2}$ and $R_{2}$ are the $\mathrm{CPE}$ and resistance for barrier layer respectively.

Figs. 3, 4 and 5 represent Nyquist and Bode plots for different immersion periods $(24,48$ and $72 \mathrm{~h}$ ) for C-HA, P-HA and LVP-HA samples respectively. The results of $24 \mathrm{~h}$ immersion are already discussed above. Further immersion up to $48 \mathrm{~h}$ in the corrosive solution, identical impedance spectra are observed and there is only slight decrease in the capacitive arc as indicated by the Nyquist plots for all the samples. Hence a very little change in $R_{\mathrm{p}}$ values is found with increasing the immersion time up to $48 \mathrm{~h}$.

The oxide coating has heterogeneities that make the capacitive behaviour better simulated by constant phase elements (CPE) than by simple capacitances $(C)$. CPE and $R_{\mathrm{p}}$ values are summarised in Table 2. $\mathrm{CPE}$ is also an important parameter in impedance studies and is defined as

$$
\mathrm{CPE}=1 /(j 2 \pi f C)^{n}
$$

where $f$ is frequency, $n$ is the frequency dispersion factor that varies from 1 to 0 . Only when $n=1, \mathrm{CPE}$ is considered to be real capacitance. Better the uniformity or corrosion resistance of the coating lower is the value of $\mathrm{CPE}^{[1,4]}$. The $\mathrm{CPE}$ values for all the samples lie between $1.48 \times 10^{-7}$ and $4.59 \times 10^{-7} \mathrm{~F} / \mathrm{cm}^{2}$. There

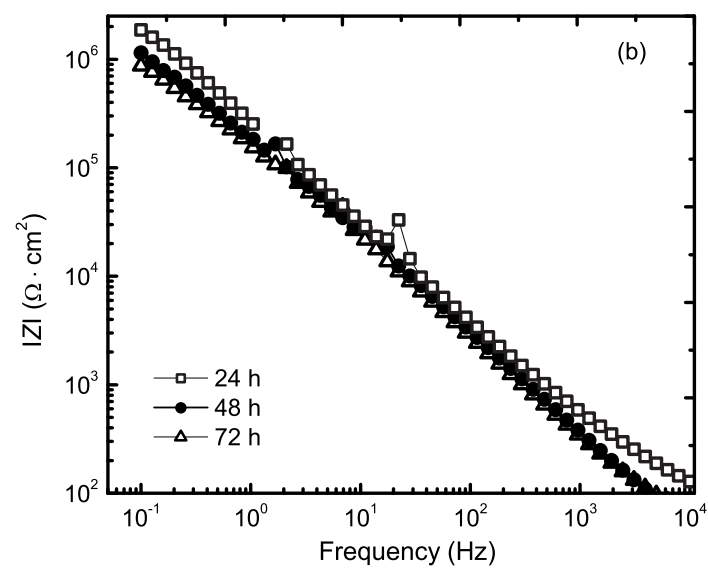

Fig. 3 Nyquist diagram (a) and Bode plots (b) for unsealed conventional hard anodised (C-HA) specimens for different immersion periods of $24 \mathrm{~h}, 48 \mathrm{~h}$ and $72 \mathrm{~h}$ 

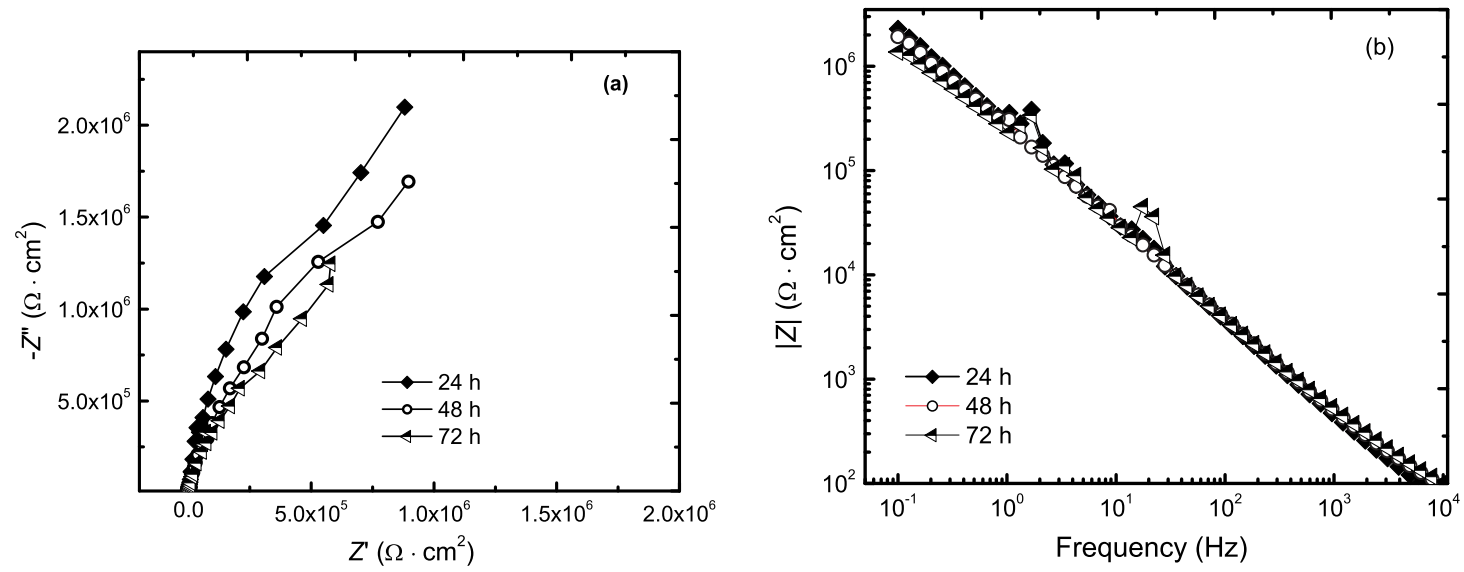

Fig. 4 Nyquist diagram (a) and Bode plots (b) for unsealed pulse hard anodised (P-HA) specimens for different immersion periods of $24 \mathrm{~h}, 48 \mathrm{~h}$ and $72 \mathrm{~h}$
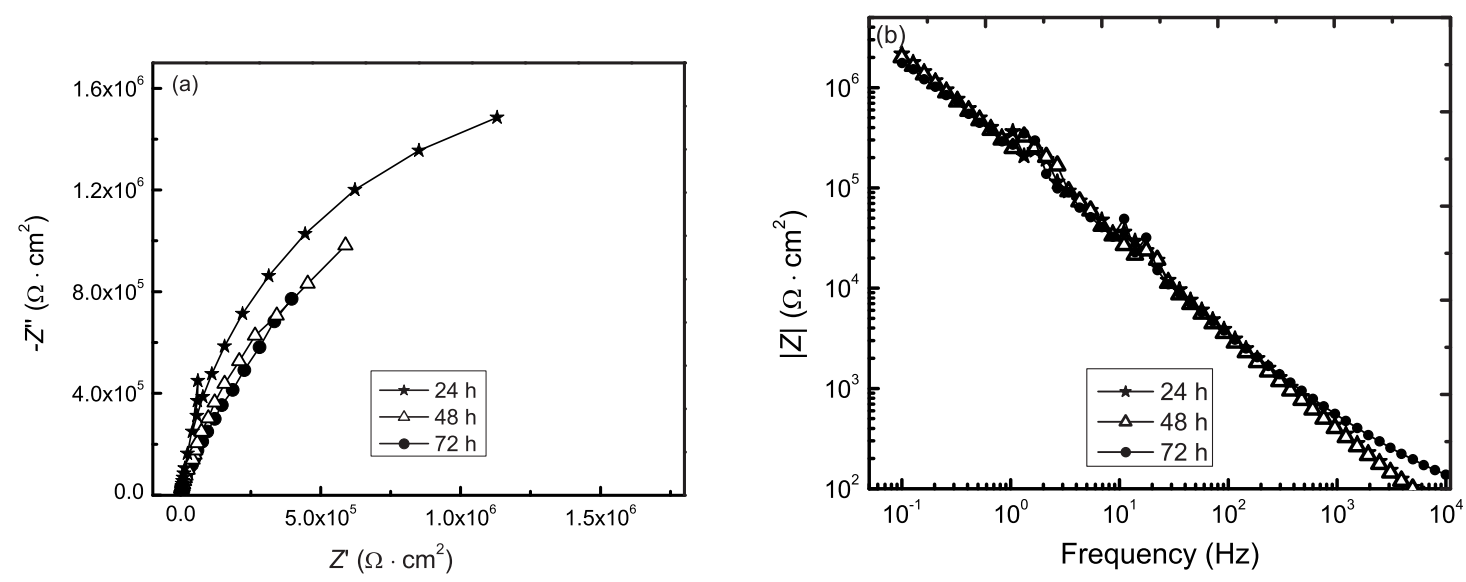

Fig. 5 Nyquist diagram (a) and Bode plots (b) for unsealed low voltage and room temperature pulse hard anodised (LVP-HA) specimens for different immersion period of $24 \mathrm{~h}, 48 \mathrm{~h}$ and $72 \mathrm{~h}$

is an increase in the values over the exposure periods. $\mathrm{C}-\mathrm{HA}$ has the $\mathrm{CPE}$ values increase from $1.48 \times 10^{-7}$ to $3.07 \times 10^{-7} \mathrm{~F} / \mathrm{cm}^{2}$. Similarly P-HA shows the CPE values to increase from $2.07 \times 10^{-7}$ to $3.51 \times 10^{-7} \mathrm{~F} / \mathrm{cm}^{2}$. LVP-HA has the same trend in which the values raise from $3.42 \times 10^{-7}$ to $4.59 \times 10^{-7} \mathrm{~F} / \mathrm{cm}^{2}$. The result reflects that uniformity of the coatings is losing slightly over the exposure periods. The decrease in impedance and increase in CPE values observed with respect to immersion time can be attributed to the slight dissolution of the porous layer.

The Bode modulus of all three types of the hard anodised samples are depicted in Figs. 3(b), 4(b) and 5 (b) which show the impedances towards low frequency are almost similar even after $48 \mathrm{~h}$ of immersion comparing to their respective $24 \mathrm{~h}$ immersion. This infers that all the hard anodised coatings do not undergo major damage. On prolonged immersion up to $72 \mathrm{~h}$, there is no drastic drop in $R_{\mathrm{p}}$ values, though some slight decrease in the values are observed due to infiltration of corrosion medium into the cracks and defects of the outer oxide layer. Fig. 6 represents the change in $R_{\mathrm{p}}$ as a function of immersion time for the unsealed samples. The slopes are gradually decreasing without steep changes which indicate that there is no rapid decrease in $R_{\mathrm{p}}$ on increasing immersion time up to $72 \mathrm{~h}$. The $R_{\mathrm{p}}$ values for C-HA decreased from $10.7 \times 10^{6}$ to $5.1 \times 10^{6} \Omega \cdot \mathrm{cm}^{2}$. P-HA has the $R_{\mathrm{p}}$ values from $7.6 \times 10^{6}$ to $4.5 \times 10^{6} \Omega \cdot \mathrm{cm}^{2}$ and $R_{\mathrm{p}}$ values for LVP-HA were found to decrease smoothly as compared to the other two. Though the values decrease over the exposure periods, but this decrease is not significant. It suggests that all three hard anodised specimens show good resistance to corrosion in which C-HA exhibits slightly better corrosion resistance than P-HA and LVP-HA.

\subsection{Polarisation result for unsealed coatings}

Since corrosion is an electrochemical phenomenon, linear polarisation can be used to provide the important information on corrosion rate, corrosion current etc. In this technique, a current-potential relationship graph is obtained by scanning the specimen through a potential range that is very close to corrosion po- 
Franco M. et al.: Acta Metall. Sin. (Engl. Lett.), 2013, 26(6), 647-656.

Table 3 Polarisation data of hard anodised specimens after exposure to $3.5 \mathrm{wt} . \% \mathrm{NaCl}$ solution

\begin{tabular}{cccccccc}
\hline Immersion time $(\mathrm{h})$ & \multicolumn{3}{c}{$E_{\text {corr }}(\mathrm{V})$} & & \multicolumn{3}{c}{$i_{\text {corr }}\left(10^{-8} \mathrm{~A} / \mathrm{cm}^{2}\right)$} \\
\cline { 2 - 4 } \cline { 6 - 8 } & C-HA & P-HA & LVP-HA & & C-HA & P-HA & LVP-HA \\
\hline 24 & -0.541 & -0.625 & -0.739 & & 1.07 & 1.11 & 2.26 \\
48 & -0.584 & -0.629 & -0.382 & & 1.18 & 1.75 & 3.19 \\
72 & -0.596 & -0.723 & -0.852 & & $2.91(0.134)$ & $3.84(0.28)$ & $4.41(0.42)$ \\
\hline
\end{tabular}

Note: values in the brackets are for the sealed speciments

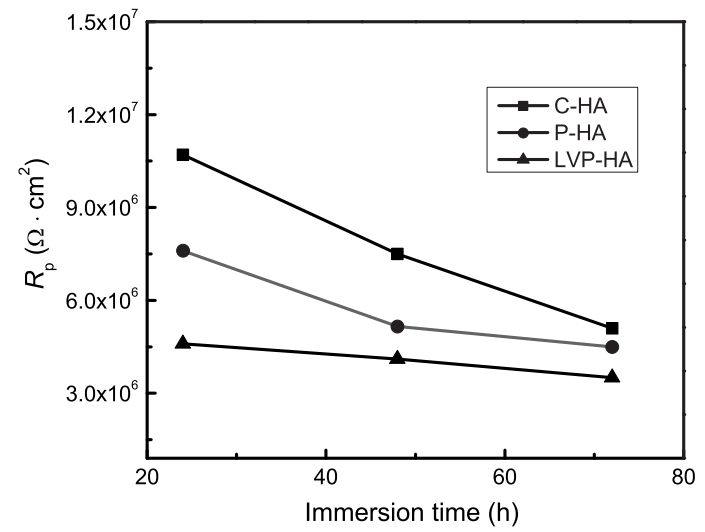

Fig. 6 Variation of polarisation resistance as a function of immersion time for unsealed samples

tential $\left(E_{\text {ocp }}\right.$ or $\left.E_{\text {corr }}\right)$. $E_{\text {ocp }}$ is the potential characteristic of the specific metal-solution with respect to a coupled reference electrode when no external current is supplied. The polarised graph is called Tafel plots ( $\lg i$ vs. potential), which comprise of anodic curve and cathodic curve. The corrosion current density, $i_{\text {corr }}$, is given by the Stern Geary equation as given below ${ }^{[27]}$ :

$$
i_{\mathrm{corr}}=\frac{\left(\beta_{\mathrm{a}}\right)\left(\beta_{\mathrm{c}}\right)}{2.303\left(\beta_{\mathrm{a}}+\beta_{\mathrm{c}}\right) R_{\mathrm{p}}}
$$

where $i_{\text {corr }}$ is corrosion current density $\left(\mathrm{A} / \mathrm{cm}^{2}\right), \beta_{\mathrm{a}}$ is anodic Tafel slope ( $\mathrm{V} /$ decade), $\beta_{\mathrm{c}}$ is cathodic Tafel slope (V/decade), $R_{\mathrm{p}}$ is polarisation resistance $(\Omega)$.

$i_{\text {corr }}$ value depicts important information regarding the corrosion behavior of the specimen surface. The larger the $i_{\text {corr }}$ value, the higher the active corrosion is.

The $E_{\text {corr }}$ of bare specimen is $-0.8664 \mathrm{~V}$ with respect to $\mathrm{Ag} / \mathrm{AgCl}$ reference electrode ${ }^{[21]}$. The hard anodised samples show positive corrosion potential when compared to bare aluminium as shown in Table 3. Thus a nobler electrode potential is observed for the hard anodic coatings compared to bare substrate. On exposure to the corrosive medium up to $72 \mathrm{~h}$, a little change in the corrosion potential is observed for all the samples. The $E_{\text {corr }}$ values for CHA are stable as compared to P-HA and LVP-HA. A slight fluctuation in the $E_{\text {corr }}$ values is recorded for $\mathrm{P}-\mathrm{HA}$ and LVP-HA over the exposure time. A more stable potential is seen for $\mathrm{C}-\mathrm{HA}$ which is towards positive potential than those of P-HA and LVPHA. The nobler $E_{\text {corr }}$ values for C-HA suggest the higher corrosion resistance of the coating. As it was
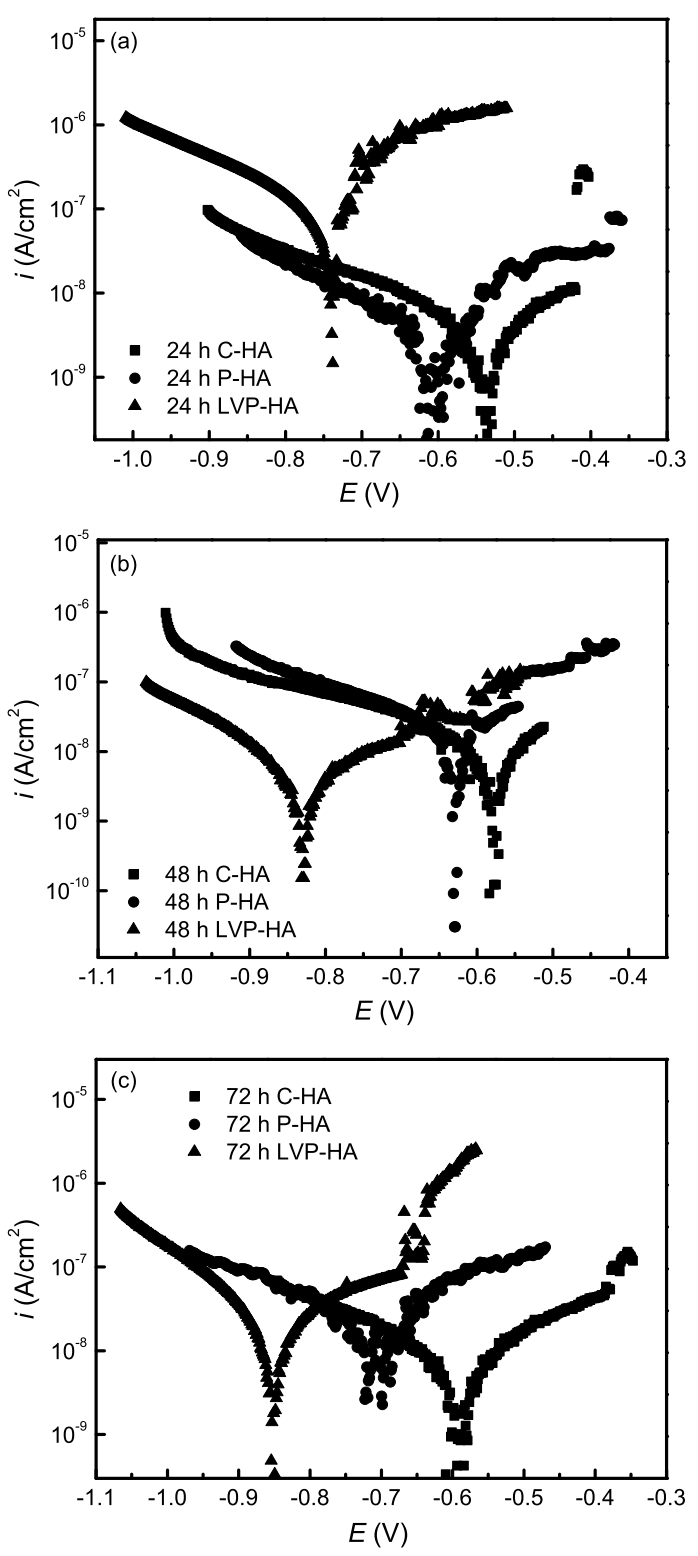

Fig. 7 Polarisation curves for different immersion periods $24 \mathrm{~h}$ (a), $48 \mathrm{~h}$ (b) and $72 \mathrm{~h}$ (c) for C-HA, P-HA and LVP-HA

emphasized by $\mathrm{Cao}^{[23]}$, the corrosion resistance cannot be evaluated by free $E_{\text {corr }}$ alone. Free $E_{\text {corr }}$ mainly describes the thermodynamic property of the material. It is scientific to evaluate the corrosion behaviour by estimating the $i_{\text {corr }}$ to support the corrosion behaviour obtained from $E_{\text {corr }}$.

Fig. 7 illustrates the Tafel plots of C-HA, P-HA and LVP-HA as a function of immersion period in 


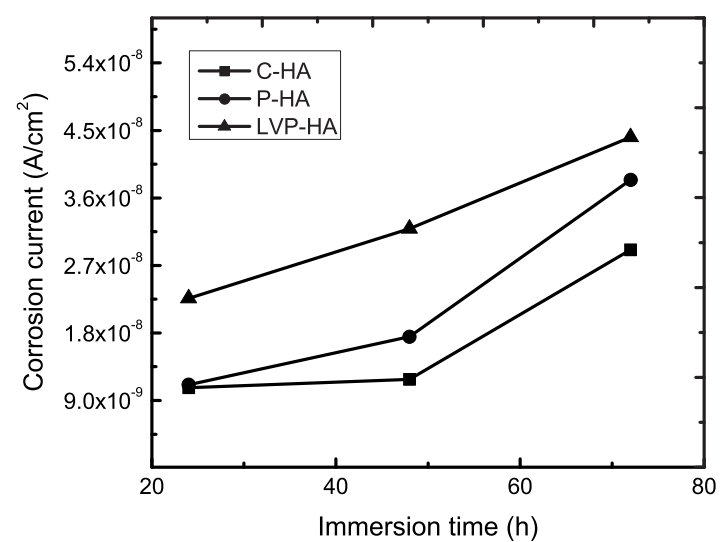

Fig. 8 Corrosion current density as a function of immersion time for unsealed hard anodised specimens

$\mathrm{NaCl}$ solution. Most of the plots show absence of sudden current increase in the polarisation curves, which confirm the non-occurrence of pitting ${ }^{[12]}$. Fig. 8 illustrates $i_{\text {corr }}$ as a function of immersion period for the hard anodised coatings, as evaluated from Fig. 7. The $i_{\text {corr }}$ values for all the unsealed hard anodised specimens are very low (in the order $10^{-8} \mathrm{~A} / \mathrm{cm}^{2}$ ) as summarised in Table 3 . The test results, i.e., $i_{\text {corr }}$ values are comparable with the values reported earlier ${ }^{[21]}$. For $24 \mathrm{~h}$ immersion, C-HA shows slightly lower $i_{\text {corr }}$ value than that of $\mathrm{P}-\mathrm{HA}$ and LVP-HA. This indicates C-HA is slightly more passive to corrosion. However, the difference in $i_{\text {corr }}$ values among three specimens is minimal. The trend is in good agreement with that observed in EIS data.

Continuation of the immersion in corrosive medium doesn't show significant change in the $i_{\text {corr }}$ value as indicated in Fig. 8. Hence, on immersion up to $72 \mathrm{~h}$, no major active corrosion has taken place. However, as expected a slight and gradual increase in corrosion on continuous exposure to corrosive medium is seen due to penetration of the medium into the pores.

\subsection{Effect of sealing on corrosion behaviour}

Fig. 9 depicts the effect of sealing on hard anodic film. The studies are conducted on unsealed and sealed hard anodized samples immersed in 3.5 wt.\% $\mathrm{NaCl}$ solution for $24 \mathrm{~h}, 48 \mathrm{~h}$ and $72 \mathrm{~h}$ duration. The dimensions of capacitive arcs have increased for the sealed hard anodized samples.

$R_{\mathrm{p}}$ values of sealed specimens have improved as compared to unsealed samples in all the cases as presented in Table 2. For C-HA, $R_{\mathrm{p}}$ values have raised from $10.7 \times 10^{6}$ to $49.5 \times 10^{6} \Omega \cdot \mathrm{cm}^{2}$. The $R_{\mathrm{p}}$ values for P-HA have also found to increase from 7.6 $\times 10^{6}$ to $27.16 \times 10^{6} \Omega \cdot \mathrm{cm}^{2}$. Similarly the $R_{\mathrm{p}}$ values for LVP-HA have enhanced from $4.6 \times 10^{6}$ to 19.18 $\times 10^{6} \Omega \cdot \mathrm{cm}^{2}$. Thus, corrosion resistance of the hard anodised samples is further enhanced on subjecting to hydrothermal sealing. A reasonable explanation
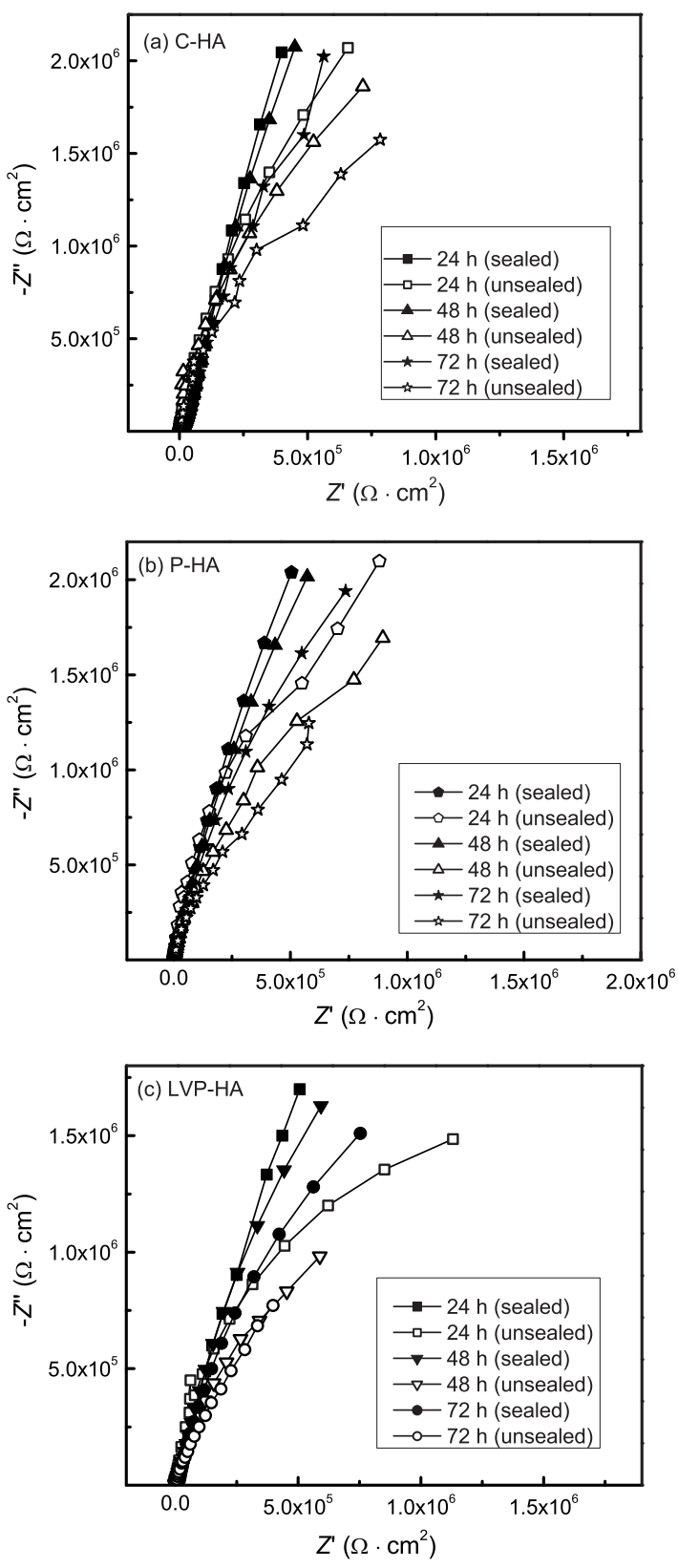

Fig. 9 Nyquist plots to illustrate the effect of sealing for C-HA (a), P-HA (b) and LVP-HA (c)

for the increase in corrosion is that during sealing process in boiling water, various forms of hydrated alumina are produced. The following sealing reaction occurs at temperature above $80{ }^{\circ} \mathrm{C}$.

$$
\mathrm{Al}_{2} \mathrm{O}_{3}+\mathrm{H}_{2} \mathrm{O} \rightarrow 2 \mathrm{AlO}(\mathrm{OH}) \text { (Boehmite) }
$$

Boehmite is the major hydrated alumina formed besides aluminium hydroxide. The hydrated alumina expands and occupies greater volume and micro-pores in the porous anodised film are filled with it ${ }^{[12,28]}$. Thus the penetration of chloride ions, water and oxygen are retarded giving rise to better corrosion resistance. The values of $i_{\text {corr }}$ for all the sealed hard anodised specimens also decrease by an order of 1 as 


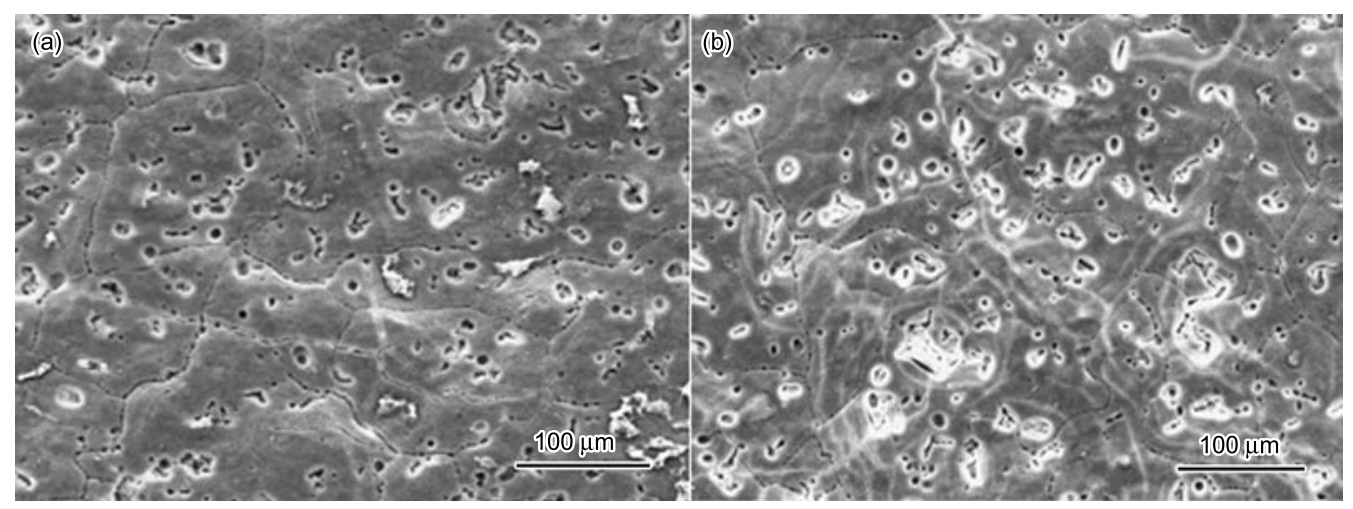

Fig. 10 SEM micrographs of sealed C-HA: (a) before immersion; (b) after immersion of $72 \mathrm{~h}$

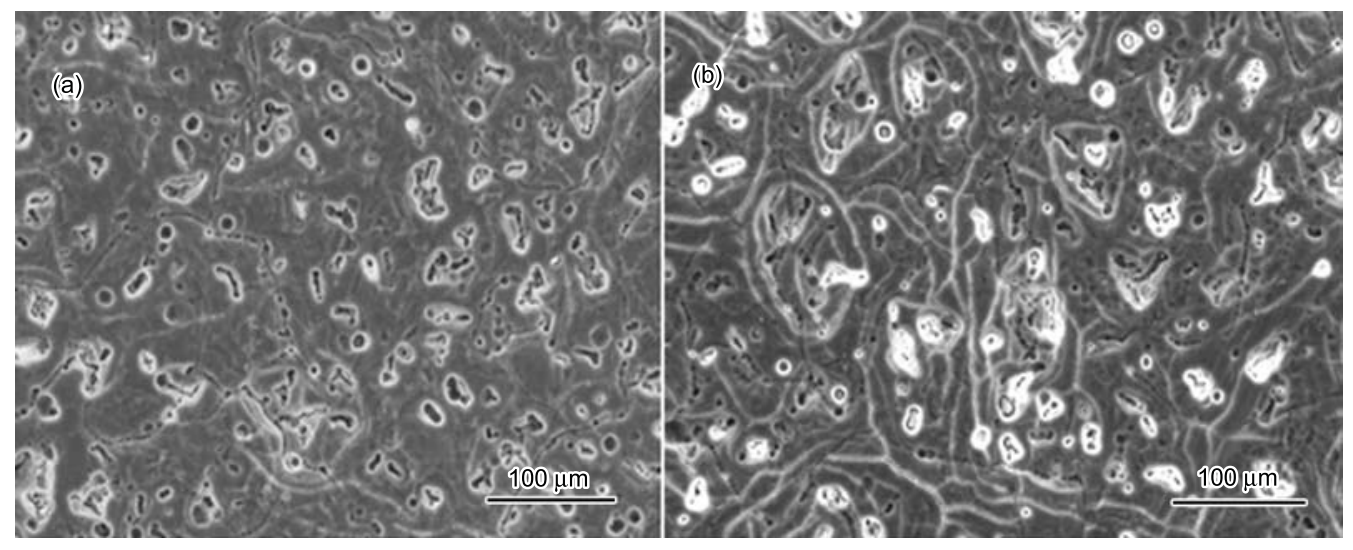

Fig. 11 SEM micrographs of sealed P-HA: (a) before immersion; (b) after immersion of $72 \mathrm{~h}$

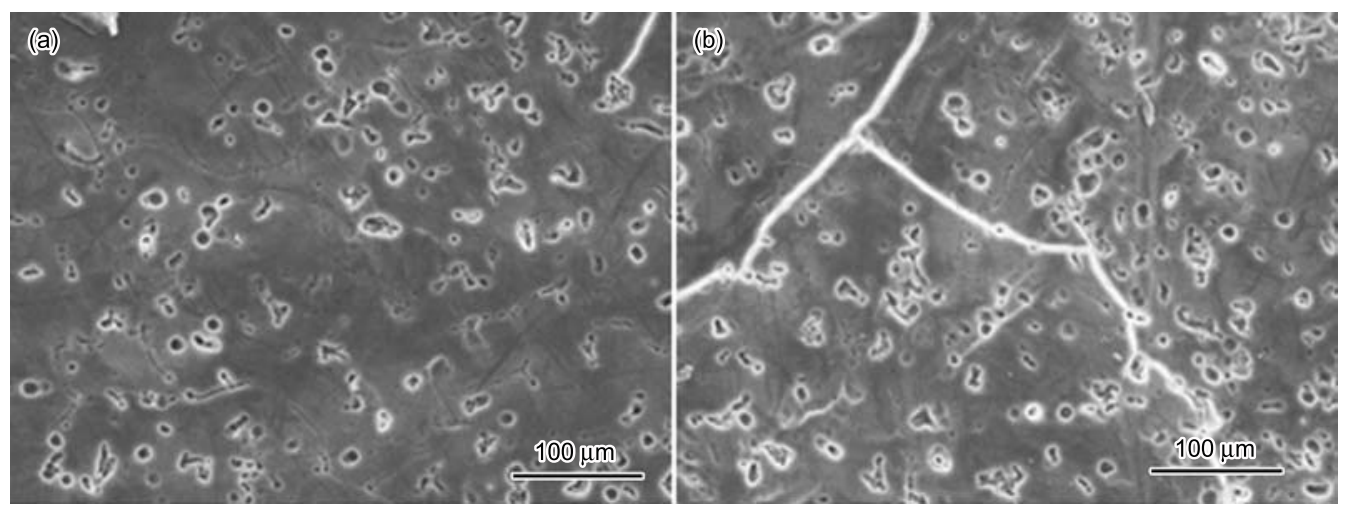

Fig. 12 SEM micrographs of sealed LVP-HA: (a) before immersion; (b) after immersion of $72 \mathrm{~h}$

given within parentheses in Table 3 . This is in a good agreement with the trend observed in impedance studies.

\subsection{Analysis of damage function}

The damage function $(D)$ is based on the change in impedance in low frequency region. It is an important parameter characteristic of corrosion behaviour ${ }^{[29,30]}$. The damage function is

$$
D=\lg \left(Z_{0} / Z_{t}\right)_{0.1 \mathrm{~Hz}}
$$

where $Z_{0}$ and $Z_{t}$ are impedance values respectively at $t=0$ and $t=t_{\text {corr }}(72 \mathrm{~h}$ in the present study). When
$D=0$, the specimen shows a perfect corrosion resistance. Increase in $D$ value shows decrease in corrosion resistance. The $D$ values of $\mathrm{C}-\mathrm{HA}, \mathrm{P}-\mathrm{HA}$ and LVP-HA under unsealed condition are $0.322,0.218$ and 0.118 respectively. This shows the change in Impedance for LVP-HA is lower on immersion upto $72 \mathrm{~h}$ as compared to that of C-HA and P-HA.

\subsection{Corrosion morphology}

Microcracks, also called crazing, which are typical and characteristic of hard anodised surfaces, are observed on all three types of as prepared hard anodic coatings (Fig. 10-12). SEM micrographs of the samples taken after immersion for $72 \mathrm{~h}$ in corrosive me- 


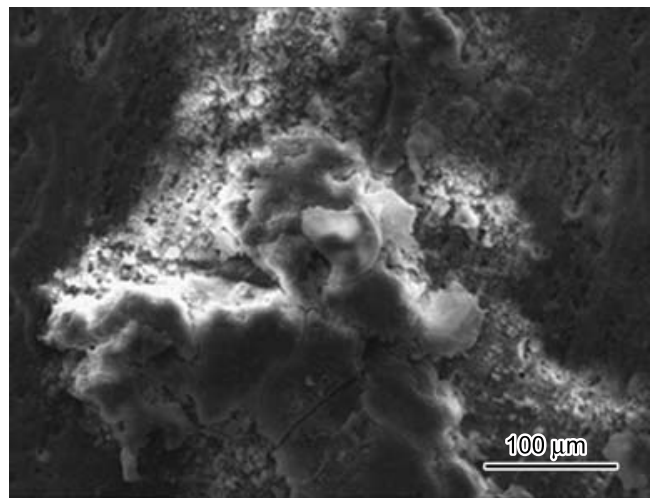

Fig. 13 SEM micrograph of a corrosion spot on unsealed C-HA after $72 \mathrm{~h}$ immersion

dia revealed initiation of corrosion around the pores. This may be due to meager penetration of corrosion solution through these micro cracks of anodic coating. LVP-HA shows more prominent micro cracks after $72 \mathrm{~h}$ of immersion than the other two. This may be the possible reason for the slight decrease in the impedance value of LVP-HA as compared to that of $\mathrm{P}-\mathrm{HA}$ and C-HA. However, major pitting corrosion is not observed in any of the three hard anodised samples.

For unsealed hard anodised specimens, few corrosion spots were observed under visual inspection as given in Fig. 13. A closer examination of the corrosion spots using SEM shows deposition of granular corrosion product. Thus it can be concluded that hydrothermal sealing of the hard anodised samples can prevent crevice corrosion and also degradation by chloride ion attack can be retarded.

\section{Conclusions}

Corrosion behaviour of three different hard anodic coatings viz, conventional, pulse and low voltage room temperature pulse hard anodisation (C-HA, P-HA, LVP-HA) on AA 6061 was investigated using electrochemical impedance spectroscopy(EIS) and linear polarisation techniques.

Very large impedance curves were obtained for all three hard anodic coatings on exposure to corrosive 3.5 wt. $\% \mathrm{NaCl}$ solution. The large dimensions of capacitive arcs and impedance values reflect the high corrosion resistance of all the samples. Though there is no significant difference in the corrosion resistance among three samples, the corrosion resistance of the unsealed specimens was found in the order C-HA $>$ PHA > LVP-HA. Further upon immersion up to $72 \mathrm{~h}$, no remarkable change in $R_{\mathrm{p}}$ values was found. This is shown by the identical impedance spectra which were recorded during the immersion periods confirming the coatings are stable and intact.

$E_{\text {corr }}$ values were generally found to be stable though there were some minor fluctuations. There were little increases in $i_{\text {corr }}$ values over the exposure periods which can be attributed to the slight dissolution of outer porous layer. SEM micrographs revealed minor degradation and corrosion had just initiated around the pores and cracks after $72 \mathrm{~h}$ of immersion. LVP-HA samples showed more prominent micro cracks after $72 \mathrm{~h}$ of immersion than the other two samples.

From the damage function analysis, $D$ is lower for LVP-HA than that of P-HA and C-HA which illustrates change of impedance as a function of immersion time is lower for LVP-HA than those of P-HA and C-HA.

After hydrothermal sealing, the $R_{\mathrm{p}}$ values for all the specimens have further improved which was shown by increase in the dimension of the impedance arc. Post sealing corrosion resistance of all three types of hard anodised samples follow the same trend as seen in the unsealed condition. In this case also there was no significant difference in the $R_{\mathrm{p}}$ values of coatings obtained with different processes. A very little change in impedance spectra were observed on exposure periods in corrosive medium. Thus, from the overall results it can be concluded that LVP-HA and P-HA have corrosion behavior comparable to that of $\mathrm{C}-\mathrm{HA}$.

\section{REFERENCES}

[1] L. Wen, Yaming Wang, Y. Zhou, J.H. Ouyang, L.X. Guo and D.C. Jia, Corros. Sci. 52 (2010) 2687.

[2] M.J. Bartolomé, J.F. del Río, E.Escudero, S.Feliu Jr., V.López, E.Otero and J.A. González, Surf. Coat. Technol. 202 (2008) 2783.

[3] Y.L. Huang, H. Shih, H.C. Huang, J. Daugherty, S. Wu, S. Ramanathan, C. Chang and F. Mansfeld, Corros. Sci. 50 (2008) 3569.

[4] X.H. Zhao, Y. Zuo, J.M. Zhao, J.P. Xiong and Y.M. Tang, Surf. Coat. Technol. 200 (2006) 6846.

[5] A.K. Sharma, H. Bhojraj, V.K. Kaila and H. Narayanamurthy, Met. Finish 95 (1997) 14.

[6] J.P.C Dasquet, D. Caillard, E. Conforto, J.P. Bonino and R. Bes, Thin Solid Films 371 (2000) 183.

[7] G.E Thompson, Thin Solid Films, 297 (1997) 192.

[8] J. Edwards, Coating and Surface Treatment Systems for Metals, vol. 38, ASM International, Finishing Publications Ltd., 1994, pp.35-40.

[9] David R. Gabe, Met. Finish. 100 (2002) 52.

[10] L.R. Krishna, A. S. Purnima and G. Sundararajan, Wear, 261 (2006) 1095.

[11] A. Rajendra, J.P. Biren, A.K. Sharma, H. Bhojraj, M.M. Nayak and K. Rajanna, Surf. Eng. 21 (2005) 193.

[12] Y. Zuo, P.H. Zhao and J.M Zhao, Surf. Coat. Technol. 166 (2003) 237.

[13] J.J. Suay, E. Gimenez, T. Rodriguez. K. Habbib and J.J. Saura, Corros. Sci. 45 (2003) 611.

[14] J.A. Gonzalez, M. Morcillo, E. Escudero, V. Lopez and E. Otero, Surf. Coat. Technol. 153 (2002) 225.

[15] V. Lopez, J.A. Gonzalez, E. Otero, E. Escudero and M. Morcillo, Surf. Coat. Technol. 153 (2002) 235.

[16] A.S. Handy, E. El-Shenawy and T. El-Bitar, Int. J. Electrochem. Sci. 1 (2006) 171. 
[17] V. Lopez, E. Otero, A. Bautista and J.A. Gonzalez, Surf. Coat. Technol. 124 (2000) 76.

[18] A. Bautista, J.A. Gonzalez and V. Lopez, Surf. Coat. Technol. 154 (2002) 49.

[19] F. Snogan, C. Blanc, G. Murkowski and N. Pebere, Surf. Coat. Technol. 154 (2002) 94.

[20] V. Lopez, J.A. Gonzalez, A. Bautista and E. Otero, Corros. Sci. 40 (1998) 693.

[21] E. Barsoukov and J.R. Macdonald, Impedance Spectroscopy, Theory, Experiment and Applications, 2nd ed., Wiley-Interscience, A John Wiley \& Sons. Inc., New Jersey, 2005, pp.469-479.

[22] C.N. Cao, Principle of Corrosion Electrochemistry, Chemistry Industry Press, Beijing, China, 2004. (in Chinese)

[23] F. Mansfeld, G. Zhang and C. Chen, Plat. Surf. Finish 84 (1997) 72.
[24] J. Liang, P. Bala Srinivasan, C. Blawert and W. Dietzel, Corros. Sci. 52 (2010) 540.

[25] F. Mansfeld, University of Southern California, Los Angeles, CA USA, Technical Report No. 26, Part no. BTR206, Issue: AB, May 1999.

[26] H.B. Zhang and Y. Zuo, Appl. Surf. Sci. 254 (2008) 4930.

[27] N.P. Wasekar, A. Jyothirmayi, L.R. Krishna and G. Sundarajan, J. Mater. Eng. Perform. 17 (2008) 708.

[28] S. Wernick, R. Pinner and P.G. Sheasby, The Surface Treatment and Finishing of Aluminium and its Alloys, 6th ed., Vol. 2, ASM International, 2001, pp.865-891.

[29] C.S. Kumar, V.S. Rao, V.S. Raja, A.K. Sharma and S.M. Mayanna, Corros. Sci. 44 (2002) 387.

[30] F. Mansfeld and M.W. Kendig, J. Electrochem. Soc. 135 (1988) 828. 